\title{
High-spin spectroscopy of natural and unnatural parity states in the mirror-pair ${ }^{45} \mathrm{~V} /{ }^{45} \mathrm{Ti}$
}

\author{
M. A. Bentley, ${ }^{1,2, *}$ C. Chandler, ${ }^{2}$ P. Bednarczyk,${ }^{3,4}$ F. Brandolini,${ }^{5}$ A. M. Bruce, ${ }^{6}$ D. Curien, ${ }^{3}$ O. Dorvaux,${ }^{3}$ \\ J. Ekman, ${ }^{7,8}$ E. Farnea, ${ }^{5}$ W. Gelletly, ${ }^{9}$ D. T. Joss, ${ }^{10}$ S. M. Lenzi, ${ }^{5}$ D. R. Napoli, ${ }^{11}$ J. Nyberg, ${ }^{12}$ C. D. O'Leary, ${ }^{1,13}$ \\ S. J. Williams, ${ }^{2,9}$ and D. D. Warner ${ }^{10}$ \\ ${ }^{1}$ Department of Physics, University of York, Heslington, York YO10 5DD, United Kingdom \\ ${ }^{2}$ School of Chemistry and Physics, Keele University, Keele, Staffordshire, ST5 5BG, United Kingdom \\ ${ }^{3}$ IReS, 23 rue du Loess, BP 28 F-67037, Strasbourg, France \\ ${ }^{4}$ Niewodniczanski Institute of Nuclear Physics, PL-31342 Krakow, Poland \\ ${ }^{5}$ Departimento di Fisica dell' Università and INFN, Sezione di Padova, I-35141 Padova, Italy \\ ${ }^{6}$ School of Engineering, University of Brighton, Brighton, BN2 4GJ, United Kingdom \\ ${ }^{7}$ Department of Physics, Lund University, S-22100 Lund, Sweden \\ ${ }^{8}$ Teknik och Samhälle, Malmö Högskola, 20506 Malmö, Sweden \\ ${ }^{9}$ School of Physics and Chemistry, University of Surrey, Guildford, Surrey, GU2 7XH, United Kingdom \\ ${ }^{10}$ CCLRC Daresbury Laboratory, Daresbury, Warrington, WA4 4AD, United Kingdom \\ ${ }^{11}$ INFN, Laboratori Nazionali di Legnaro, Italy \\ ${ }^{12}$ Department of Radiation Sciences, Uppsala University, Uppsala, Sweden \\ ${ }^{13}$ Ultra Electronics, Armitage Road, Rugeley, Staffordshire WS15 1DR, United Kingdom
}

(Received 28 September 2005; published 8 February 2006)

\begin{abstract}
High-spin states in the proton-rich nucleus ${ }^{45} \mathrm{~V}$ have been identified for the first time. A comprehensive $\gamma$-ray decay scheme has been established following an experiment performed at the Vivitron accelerator at IReS Strasbourg by using the Euroball $\gamma$-ray detector array coupled to the Neutron Wall and Euclides detector arrays. The natural (negative-) parity scheme is identified up to the $f_{7 / 2}$ band termination in addition to a positive-parity collective structure based on a $d_{3 / 2}$ particle-hole excitation. Comparison of this scheme with that of the mirror partner, ${ }^{45} \mathrm{Ti}$, has yielded detailed information on the variation of Coulomb energy as a function of excitation energy and angular momentum. This is the first time that such an analysis has been performed for a collective structure built on a cross-shell excitation. Comparison of the observed Coulomb energies with those predicted by large-scale shell-model calculations is presented. In this case, unusually, the calculations do not fare as well as for heavier nuclei in the shell. In addition, stark differences between the two nuclei are observed for the decay intensities of the parity-changing $E 1$ decays that de-excite the positive-parity deformed bands.
\end{abstract}

DOI: 10.1103/PhysRevC.73.024304

PACS number(s): 21.10.Hw, 23.20.Lv, 27.40.+z, 21.10.Sf

\section{INTRODUCTION}

One of the most fundamental symmetries in Nature is the exchange symmetry between neutrons and protons in the nucleus, which reflects the approximate charge symmetry and charge independence of the attractive nucleon-nucleon interaction. Although it is now known (see, e.g. [1]) that these two symmetries are only approximate, they retain enough validity to result in startling symmetries in nuclear behavior, which can be readily observed in the study of isobaric multiplets. These symmetries lead naturally to the use of the isospin quantum number, $T$, one of the most powerful and elegant concepts in nuclear physics. States of the same isospin in a mass multiplet (i.e., isobaric analog states, IAS) will be degenerate if electromagnetic effects are ignored and if charge symmetry and/or change independence are assumed. Of course, in nuclei, the Coulomb force between protons breaks the symmetry and lifts the degeneracy of the IAS. The simplest case to consider is that of a pair of mirror nuclei $(\mathrm{A}, \mathrm{B})$ with $T_{z}=(N-Z) / 2= \pm \frac{1}{2}$ such that $Z_{A}=N_{A}+1=N_{B}$

*Electronic address: mab503@york.ac.uk and $Z_{B}=N_{B}-1=N_{A}$. The $T_{z}= \pm \frac{1}{2}$ pair discussed here, ${ }_{23}^{45} \mathrm{~V}_{22} /{ }_{22}^{45} \mathrm{Ti}_{23}$, is one such example. In such mirror pairs the energy difference between excited states-termed the mirror energy differences, MED — can be established through modern spectroscopic techniques and can yield direct information on Coulomb effects if the charge symmetry of the strong nuclear force is assumed.

Over the past decade, advances in experimental techniques-specifically the development of large $\gamma$-ray spectrometers coupled to sensitive channel-selection deviceshave afforded the opportunity to study proton-rich nuclei in detail up to $A \approx 60$. In the $f_{7 / 2}$ shell between ${ }^{40} \mathrm{Ca}$ and ${ }^{56} \mathrm{Ni}$, this has allowed the detailed spectroscopy of essentially all the $T_{z}= \pm \frac{1}{2}$ mirror pairs [2-11] and the even- $A T=1$ isobaric triplets [12-18]. In all of these cases, the energy differences between excited states have been extracted and interpreted in terms of Coulomb effects. One of the real surprises is that the quantitative analysis of Coulomb energies by this approach has yielded far more reliable and subtle information on nuclear structure phenomena than had been previously supposed. It is particularly surprising given the long-established Nolen-Schiffer anomaly [19], which refers to the large discrepancy consistently found when attempting 
to reproduce the binding energy differences of the ground states of mirror nuclei. A key advantage of performing these studies in the $f_{7 / 2}$ shell comes from the development of the large-scale $p f$-shell-model calculations, based on the code described in Ref. [20], that are available in this mass region and that have been shown to be extremely reliable. The work on isobaric analog nuclei has prompted modifications to the shell-model calculations to include both multipole (e.g. [7]) and monopole (e.g. [15,21]) Coulomb terms. Thus this work provides an extremely stringent test of this important model and, conversely, the reliability of the model provides a valuable tool in the interpretation of the measured Coulomb energies.

The relative isolation of the $f_{7 / 2}$ shell from other major orbits results in yrast structures whose state wave functions are dominated by pure $\left(f_{\frac{7}{2}}\right)^{n}$ configurations (e.g. [22]), where $n$ is the number of $f_{7 / 2}$ particles outside ${ }^{40} \mathrm{Ca}$. This, in turn, means that the evolution of Coulomb energies along the yrast line can be interpreted consistently without significant changes in configuration. It has now been demonstrated both experimentally (e.g. $[2,6,7,10]$ ) and theoretically (e.g. $[8,15,21]$ ) that the largest angular-momentum-dependent effect on the Coulomb energy in this mass region arises from the recoupling of pairs of $f_{\frac{7}{2}}$ protons from $J=0$ to $J=6$. In addition, it has been possible to relate smaller variations in the MED to other specific spin-dependent phenomena, such as changes in radius and/or deformation as a function of spin [7,15,21].

To date, all the analysis of MED for nuclei in the $f_{7 / 2}$ shell has been restricted to the natural parity structures dominated by $\left(f_{7 / 2}\right)^{n}$ configurations. However, nonyrast unnatural parity states, which are based on one-particle-one-hole excitations from the $d_{3 / 2}$ orbital below ${ }^{40} \mathrm{Ca}$, are common in this region. The Nilsson diagrams in the region show that particlehole excitations from $d_{3 / 2}$ are expected to have a prolate deformation-driving effect. The resulting strongly coupled deformed collective structures built on low-lying $J^{\pi}=$ $\frac{3}{2}^{+}$states have been established in a number of light $f_{7 / 2}$ cases, such as the $T_{z}=+\frac{1}{2}$ nuclei ${ }^{47} \mathrm{~V}$ [23] and ${ }^{49} \mathrm{Cr}$ [24]. As yet, there has been no analysis of Coulomb effects associated with these structures through studies of the analog sequences in the less accessible $T_{z}=-\frac{1}{2}$ mirror partners.

In this paper we present, for the first time to our knowledge, an extensive level scheme of the $T_{z}=-\frac{1}{2}$ nucleus ${ }^{45} \mathrm{~V}$. Before this work some low-lying states of both parities had been identified by Gronemeyer et al. [25]. The current work identifies the negative-parity yrast structure up to the $f_{7 / 2}$ band-termination state. In addition, the positive-parity structure based on the $d_{3 / 2}$ particle-hole excitation is extended up to high spins. MED have been established, and a detailed analysis of the Coulomb effects for both structures is presented and supported by the predictions of the large-scale shell-model calculations. In addition, we show evidence for an anomaly in the comparison of the $\gamma$-ray intensties depopulating the positive-parity structures.

\section{EXPERIMENTAL DETAILS}

The experiment was performed by using the Vivitron accelerator at IReS Strasbourg. A ${ }^{24} \mathrm{Mg}$ beam was accelerated to
$83 \mathrm{MeV}$ and impinged on a $500 \mu \mathrm{g} / \mathrm{cm}^{2}{ }^{24} \mathrm{Mg}$ self-supporting target. The $T_{z}= \pm \frac{1}{2}$ mirror pair, ${ }^{45} \mathrm{~V}$ and ${ }^{45} \mathrm{Ti}$, were then produced in a fusion-evaporation reaction through the $1 p 2 n$ and $2 p 1 n$ evaporation channels, respectively. Gamma rays from the reaction were detected in the Euroball array [26,27] which, at the time, consisted of 26 Clover detectors and 15 Cluster detectors, each containing 4 or 7 individual hyperpure Ge crystals, respectively-a total of 209 individual detector elements. Each individual Clover and Cluster detector unit was Compton suppressed, and within each unit $\gamma$-ray add-back routines were applied to increase the total photopeak efficiency of the array for high-energy transitions.

The predicted cross section for ${ }^{45} \mathrm{~V}$ is expected to be of the order of $1 \mathrm{mb}$-around 100 times smaller than that of the mirror nucleus ${ }^{45} \mathrm{Ti}$. Given this, and the near-identicality of the $\gamma$-ray transitions expected for the mirror pair, additional and unambiguous channel selection was necessary. To achieve this, the evaporated particles were recorded in two ancillary devices. The Euclides array [28] was used to record protons and alpha particles emitted in the reaction. Euclides consisted of 40 $\Delta E-E \mathrm{Si}$ detector telescopes arranged in five rings. Each of the five detectors in the most forward ring was electrically segmented into four parts, making a total of 55 effective telescopes in the whole array. Two-dimensional gates on the individual $\Delta E-E$ plots were used to discriminate between emitted alpha particles and protons. Of particular importance in this experiment is the identification of the number of neutrons emitted. For this purpose, the Euroball Neutron Wall [29] was used, which consisted of 50 individual detectors, containing BC501A liquid scintillators, covering a solid angle of $\approx 1 \pi$ at forward angles and located at an average distance of $\approx 0.5 \mathrm{~m}$ from the target position. Discrimination between $\gamma$ rays and neutrons was achieved by using a combination of time-of-flight (TOF) and pulse-shape discrimination, the latter utilizing the zero-crossover time (ZCO) of a bipolar amplified pulse. Discrimination was therefore achieved by using twodimensional gates on individual TOF-ZCO matrices. For this reaction and analysis procedure, the total efficiency for single-neutron detection with this array was found to be $15 \%$. The experimental trigger was defined when one neutron had been preidentified in hardware and at least one clean $\gamma$ ray had been recorded in Euroball, or when three $\gamma$ rays or more had been detected. The Euclides data was not specifically included in the trigger.

In the offline analysis the energy and angle information from the Euclides and Neutron Wall detector elements for each evaporated particle were used to perform a kinematic reconstruction of each event. This allowed the recoil vector of the final nucleus to be determined, permitting a more accurate $\gamma$-ray Doppler correction. This then reduces the Doppler broadening arising from the recoil cone.

\section{DATA ANALYSIS AND RESULTS}

In an attempt to unambiguously assign $\gamma$ rays to the weak reaction channel of interest, ${ }^{45} \mathrm{~V}$, singles $\gamma$-ray spectra were generated in coincidence with different combinations of evaporated particles. One such spectrum is found in Fig. 1(a), which 


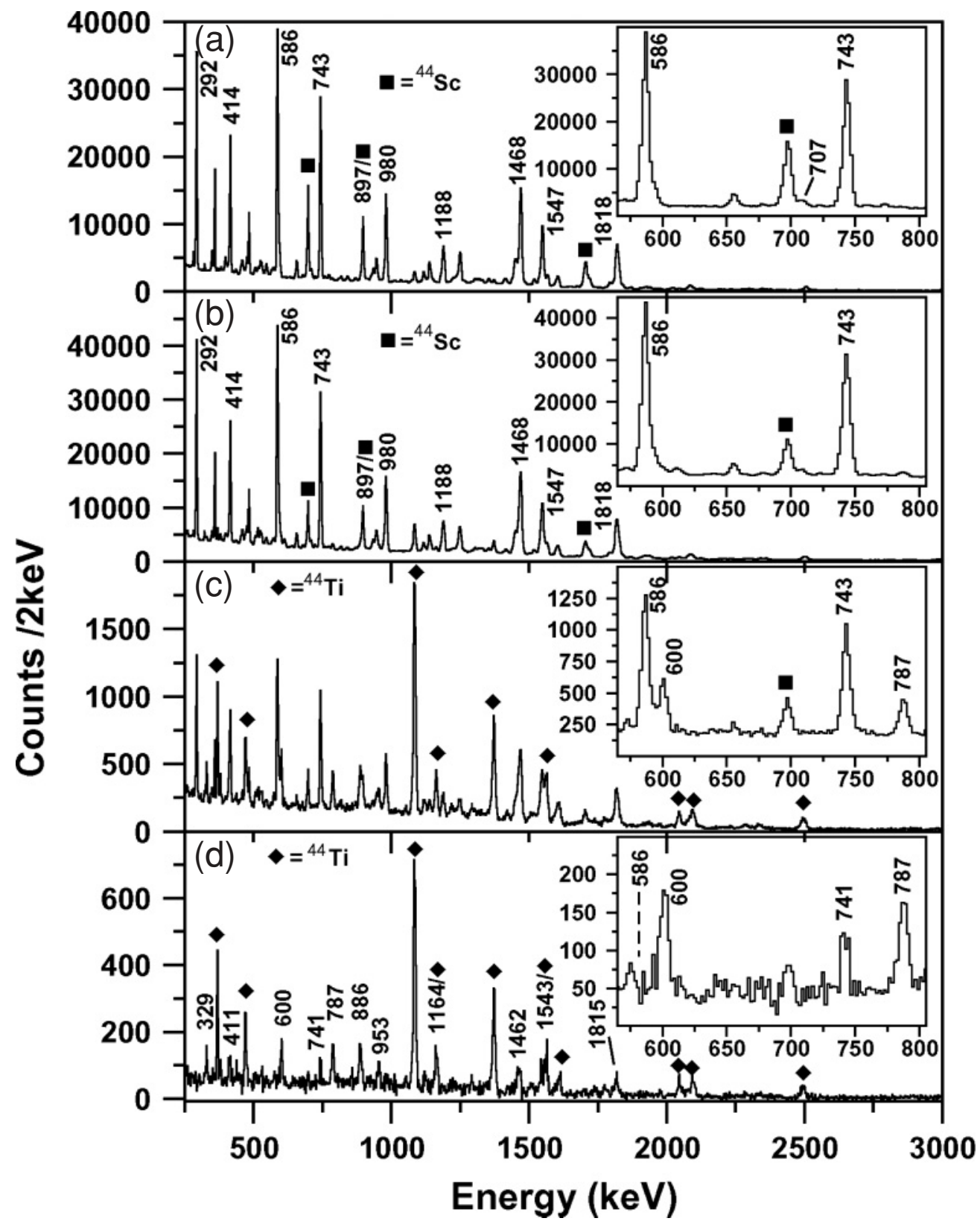

FIG. 1. Singles $\gamma$-ray spectra created with different conditions placed on the Euclides and Neutron Wall detectors. (a) Spectrum recorded when two protons and one neutron were detected. The numerically labeled peaks all originate from ${ }^{45} \mathrm{Ti}$ (the $2 p 1 n$ channel) and those labeled with the filled squares correspond to ${ }^{44} \mathrm{Sc}$ - the $3 p 1 n$ channel. The $2 p 2 n$ channel $\left({ }^{44} \mathrm{Ti}\right)$ is also present, but too weak to be labeled. (b) Spectrum recorded when two neutrons were detected. The labeling scheme is the same as (a). (c) Same spectrum as (b), but having also applied the nearestneighbor rejection procedure (see text). The peaks labeled with filled diamonds originate from the $2 p 2 n$ channel, ${ }^{44}$ Ti. (d) The clean two-neutrongated spectrum. The same as (c) but with an additional time-of-flight analysis applied (see text). The numerically labeled transitions are assigned to ${ }^{45} \mathrm{~V}$.

shows $\gamma$ rays recorded in coincidence with two protons and one neutron. As expected, the $2 p 1 n$ channel ${ }^{45} \mathrm{Ti}$ dominates, with all the labeled peaks corresponding to known [30] transitions in ${ }^{45} \mathrm{Ti}$. Also present, and marked in this spectrum, are transitions from the $3 p 1 n$ channel, ${ }^{44} \mathrm{Sc}$, where one of the three protons emitted was not detected by the Euclides array. To produce a similar spectrum for ${ }^{45} \mathrm{~V}$, the $1 p 2 n$ channel, a $2 n$-gated spectrum was produced (gating on the proton is not helpful, as all major channels have at least one proton emitted). This spectrum, requiring that two separate neutron detectors record a neutron, is shown in Fig. 1(b). As can be seen, the spectrum is virtually identical to the $2 p 1 n$-gated spectrum of Fig. 1(a). This is because when two neutron detectors record neutrons in most cases this corresponds to one scattered neutron recorded twice. To alleviate this, a nearest-neighbor-rejection procedure was employed. In this method, particular pair combinations were rejected based on their physical proximity. To determine the acceptable separation of detectors, a correlation analysis 


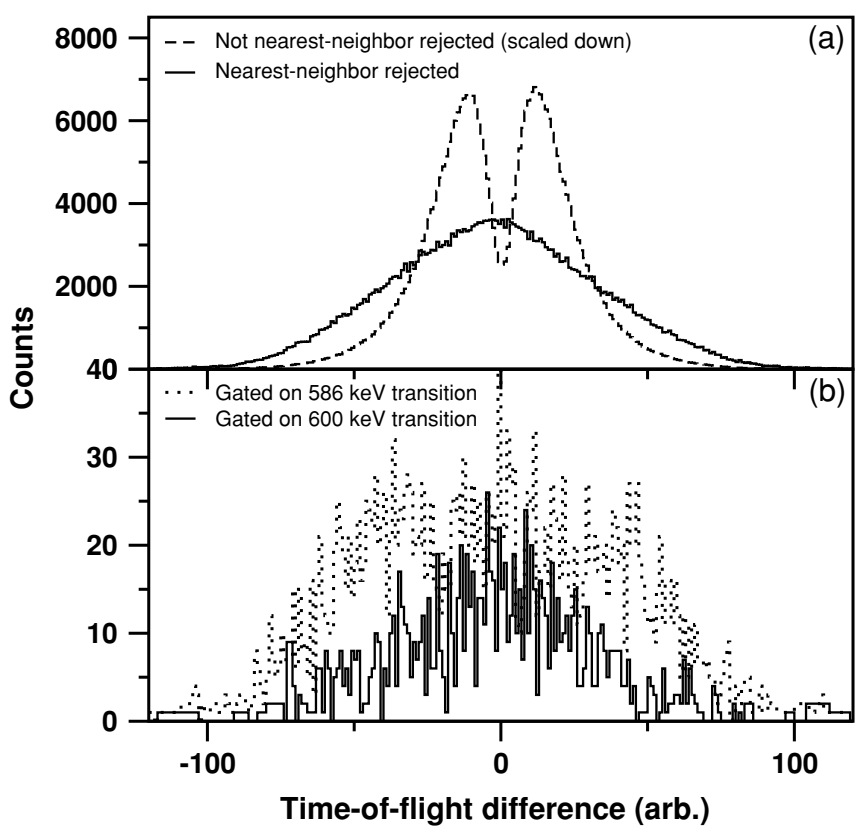

FIG. 2. Neutron time-of-flight difference, in arbitrary units, when two neutron detectors fired. (a) Dashed curve, all two-neutron events. Solid curve, two-neutron events after the nearest-neighbor rejection procedure had been applied. (b) Solid curve, in coincidence with the $600 \mathrm{keV}$ transition in ${ }^{45} \mathrm{~V}$. Dashed curve, in coincidence with the $586 \mathrm{keV}$ transition in ${ }^{45} \mathrm{Ti}$.

was performed in which the coincidence frequency of each pair of detectors was analysed. Specific pair combinations were rejected if the coincidence frequency was above a certain level. The spectrum resulting from this procedure is shown in Fig. 1(c). Although some of the ${ }^{45} \mathrm{Ti}$ remains, the spectrum is now dominated by the $2 p 2 n$ channel ${ }^{44} \mathrm{Ti}-$ the strongest of the $2 n$ channels.

To identify the ${ }^{45} \mathrm{~V}$ transitions, the remainder of the scattered $1 n$ events need to be removed. This was achieved by an additional time-of-flight (TOF) analysis suggested by Ljungvall et al. [31], where the difference in TOF between the two neutrons in the $2 n$ event is analyzed. For genuine $2 n$ events the TOF should be approximately the same for each neutron, and the TOF difference should be zero. For scattered $1 n$ events, now that near-neighbor coincidences have been removed, the TOF difference should be nonzero. This can clearly be seen in Fig. 2(a), where the TOF difference is shown for all $2 n$ events (i.e., mostly scattered $1 n$ events) and for events where the nearest-neighbor-rejection analysis has been applied (i.e., mostly real $2 n$ events). The final pure $2 n$ spectrum was produced by gating on the central region of the TOF-difference spectrum, with appropriate background subtractions. The result is shown in Fig. 1(d). Close examination of the spectrum shows that all of the $1 n$ transitions have been removed. For example, the insets in Fig. 1(a) to 1(c) all show the strong $586 \mathrm{keV}$ transition in ${ }^{45} \mathrm{Ti}$, which is completely gone in the inset of Fig. 1(d). Thus we can be confident that only genuine $2 n$ events remain.

Figure 1(d) is, as expected, dominated by transitions from the $2 p 2 n$ channel, ${ }^{44} \mathrm{Ti}$, but should also contain the transitions from ${ }^{45} \mathrm{~V}$. From the work of Gronemeyer et al. [25], a few low-lying states and $\gamma$ transitions had previously been identified. The only known natural parity states were two nonyrast states with $J^{\pi}=\frac{3}{2}^{-}$and $\frac{5}{2}^{-}$, in addition to the $J^{\pi}=\frac{7}{2}^{-}$ground state. The $J^{\pi}=\frac{3}{2}^{+}, \frac{5}{2}^{+}$, and $\frac{7}{2}^{+}$members of the band built on the $d_{3 / 2}$ intruder level had also been identified. The 329 and $411 \mathrm{keV}$ decays from the $\frac{3}{2}^{+}$and $\frac{5}{2}^{+}$states, respectively, can be seen in Fig. 1(d). In addition to these, and the well-known transitions in ${ }^{44} \mathrm{Ti}$, a number of other peaks can be identified that have energies very close to those in ${ }^{45} \mathrm{Ti}$, and these we assign to ${ }^{45} \mathrm{~V}$. Each of the labeled transitions in the ${ }^{45} \mathrm{Ti}$ spectrum in Fig. 1(a) has a corresponding (mirrored) transition in ${ }^{45} \mathrm{~V}$, which is marked in Fig. 1(d). The strongest suggested ${ }^{45} \mathrm{~V}$ transition is the $600 \mathrm{keV}$ transition, presumed to be the mirror of the $586 \mathrm{keV} \mathrm{J}{ }^{\pi}=\frac{17}{2}^{-} \rightarrow \frac{15}{2}^{-}$transition in ${ }^{45}$ Ti. Figure 2(b) shows the TOF-difference spectrum for these two transitions. Comparison with Fig. 2(a) clearly shows that the $600 \mathrm{keV}$ transition is associated with the emission of two neutrons.

Figure 3 shows the level schemes of the $A=45$ mirror pair, on the left-hand side of which is the scheme of ${ }^{45} \mathrm{~V}$ deduced from this work. On the right-hand side is shown a portion of the ${ }^{45} \mathrm{Ti}$ level scheme, based on the comprehensive published level schemes of Bednarczyk et al. [30,32]. The current work confirms the published schemes. The placement and ordering of the $\gamma$ rays in the new scheme for ${ }^{45} \mathrm{~V}$ was achieved through a detailed 2D and 3D $\gamma$-ray analysis, described below. Owing to the very close proximity of the $\gamma$-ray energies of the transitions in the mirror pair, production of clean $\gamma$-ray gated spectra for ${ }^{45} \mathrm{~V}$ was extremely difficult, with only the $329 \mathrm{keV}$ transition providing a clean gate. In addition, the low beam intensity $(\leqslant 1 \mathrm{pnA})$ of the difficult ${ }^{24} \mathrm{Mg}$ beam, coupled with the low cross section for production of ${ }^{45} \mathrm{~V}$, limited the statistics obtained. Thus, a clean two-neutron-gated $\gamma-\gamma$ analysis was not possible. These issues conspired to such an extent that an angular correlation analysis was not possible in this case. However, given that the placement of the $\gamma$ rays in the scheme has been confirmed through a $\gamma$-ray coincidence analysis (see below), we can confidently assign the multipolarities of the $\gamma$ rays, and hence the spins and parities of the states, by mirror symmetry arguments based on comparison with the well-known [30] ${ }^{45} \mathrm{Ti}$ scheme. There is no evidence to date that suggests that this method is not completely reliable, and we therefore quote the spins and parities as unambiguous assignments, based on the well-established assignments of the analog states in the mirror partner ${ }^{45} \mathrm{Ti}$ [30]. The measured energies and intensities of the $\gamma$ rays, and the state energy, spin, and parity assignments are listed in Table I. The remarkable similarity in the decay intensities of the states (with one notable exception, which will be discussed later) can be seen in the table.

No $\gamma$ decays in the yrast $f_{7 / 2}$ (negative parity) band in ${ }^{45} \mathrm{~V}$ have been observed previously. The members of this band were identified first in Fig. 1(d) by comparison with known transitions in ${ }^{45} \mathrm{Ti}$ and then confirmed through $\gamma$-ray coincidence analysis. In this way the structure shown in Fig. 3 up to the $J^{\pi}=\frac{27}{2}^{-}$band-termination state was established. 

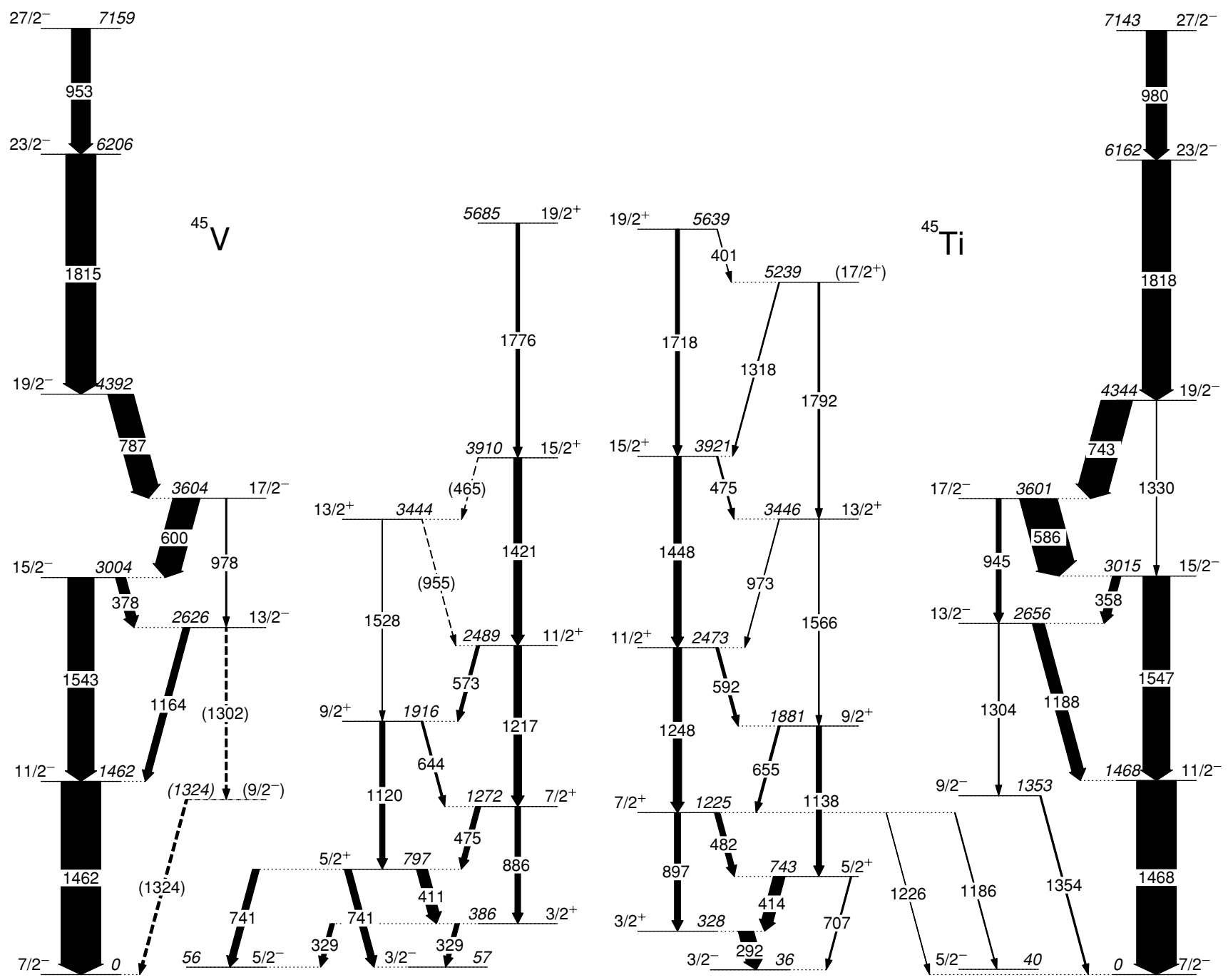

FIG. 3. Deduced energy level scheme (left) for ${ }^{45} \mathrm{~V}$ from this work. Spins and parities were assigned through mirror-symmetry arguments, and the widths of the arrows are proportional to the relative intensity of the $\gamma$-ray transitions. On the right is a portion of the ${ }^{45} \mathrm{Ti}$ scheme, taken from Ref. [30] and confirmed in the present work.

The $\gamma-\gamma$ coincidence (doubles) analysis was undertaken with a $\gamma-\gamma$ matrix incremented on the condition that at least one neutron was detected. Figures 4(a) and 4(b) show spectra generated in coincidence with the $787 \mathrm{keV}\left(\frac{19}{2}^{-} \rightarrow \frac{17}{2}^{-}\right.$) transition in ${ }^{45} \mathrm{~V}$ and the equivalent $743 \mathrm{keV}$ transition in ${ }^{45} \mathrm{Ti}$. The symmetry between the spectra is obvious, and the sequence up to the band termination is clearly seen. A triple $\gamma$-ray coincidence analysis was also undertaken to confirm the ordering of the ${ }^{45} \mathrm{~V}$ scheme and to reduce contamination in the spectra. Figure 5(a) shows a spectrum generated in coincidence with both the $787 \mathrm{keV}$ transition and any one of the 378,600 , or $953 \mathrm{keV}$ yrast-band transitions in ${ }^{45} \mathrm{~V}$. Figure $5(\mathrm{~b})$ shows the equivalent spectrum produced by using the mirrored transitions in ${ }^{45} \mathrm{Ti}$. Again the sequence is confirmed, and some of the weaker transitions are seen, such as those populating and de-exciting the $J^{\pi}=\frac{9}{2}^{-}$state (although the order of these two was not determined). It should be noted that the ratio of the intensities between the equivalent spectra for ${ }^{45} \mathrm{Ti}$ and ${ }^{45} \mathrm{~V}$ is always around the factor of 100 expected from the relative cross sections. This can be seen clearly in Figs. 4, 5 , and 6 .

The positive-parity sequence in ${ }^{45} \mathrm{~V}$ is based on the $d_{3 / 2}$ $J^{\pi}=\frac{3}{2}^{+}$intruder band head at $386.1 \mathrm{keV}$ [25]. Also observed in Ref. [25] were the $J^{\pi}=\frac{5}{2}^{+}$and $\frac{7}{2}^{+}$states of this sequence. All the other states shown in this sequence in Fig. 3 up to $J^{\pi}=\frac{19}{2}^{+}$are new. Gronemeyer et al. [25] identified two $E 1$ transitions de-exciting the $J^{\pi}=\frac{3}{2}^{+}$state. These populate the nonyrast $J^{\pi}=\frac{3}{2}^{-}$and $J^{\pi}=\frac{5}{2}^{-}$states at 57.2 and $56.4 \mathrm{keV}$ via 328.9 and $329.7 \mathrm{keV}$ transitions. In the current work, these two cannot be distinguished, so Fig. 3 and Table I quote the measured average energy (329.1 keV), and the intensity is distributed equally between them. The same approach is taken for the $741 \mathrm{keV}$ transition decaying to these two near-degenerate states from the $J^{\pi}=\frac{5}{2}^{+}$level (although Gronemeyer et al. show this decaying exclusively to the $J^{\pi}=\frac{5}{2}^{-}$state). The $1 n$-gated $\gamma-\gamma$ matrix was also used for a doubles analysis of this sequence of states. Figures 4(c) 
TABLE I. Assigned spin, parity and excitation energy for excited states and transition energy and relative intensity for $\gamma$ decays for ${ }^{45} \mathrm{~V}$ and ${ }^{45} \mathrm{Ti}$. Intensities are quoted relative to the strong $11 / 2^{-}$to $7 / 2^{-}$yrast transition.

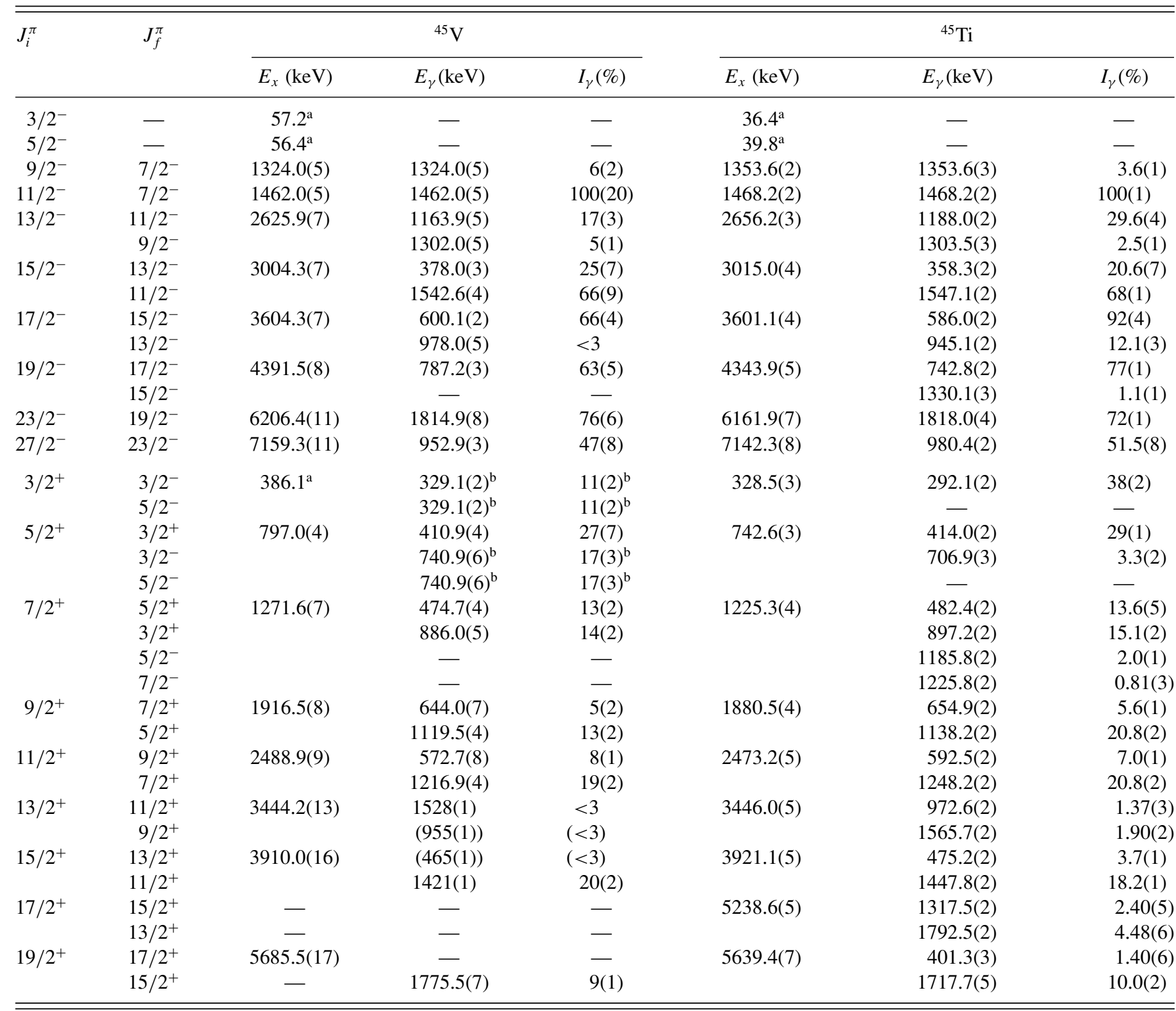

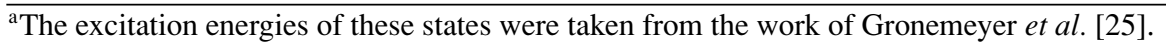

${ }^{\mathrm{b}}$ For these 329 and $741 \mathrm{keV}$ transitions in ${ }^{45} \mathrm{~V}$ the final state cannot be determined in this work (see text). Thus the average $\gamma$-ray energy is recorded and the intensity split equally between the two possible decay paths.

and 4(d) show spectra generated in coincidence with the $329 \mathrm{keV}$ transition in ${ }^{45} \mathrm{~V}$ and the $292 \mathrm{keV}$ equivalent transition in ${ }^{45} \mathrm{Ti}$. Again, all the transitions in the sequence up to $J^{\pi}=\frac{19}{2}^{+}$are seen. This is confirmed in the triples coincidence analysis. Figure 5(c) shows a spectrum generated in coincidence with both the $329 \mathrm{keV}$ transition and any of the $411,475,886$, or $1217 \mathrm{keV}$ transition in the positive-parity band in ${ }^{45} \mathrm{~V}$. Figure $5(\mathrm{~d})$ shows the equivalent spectrum produced by using the mirrored transitions in ${ }^{45} \mathrm{Ti}$.

An example of how weak transitions were placed in the level scheme is shown in Fig. 6, which shows double-gated spectra for the positive-parity structure. These spectra firmly place the $1776 \mathrm{keV}$ transition to be the $J^{\pi}=\frac{19}{2}^{+} \rightarrow \frac{15}{2}^{+}$member of the sequence (and not the $J^{\pi}=\frac{17}{2}^{+} \rightarrow \frac{13}{2}^{+}$, as mirror symmetry of transition energies might suggest). Figures 6(a) and 6(c) show spectra for ${ }^{45} \mathrm{~V}$ double-gated on both the $329 \mathrm{keV}$ transition and the (a) $1217 \mathrm{keV}$ or (c) $1776 \mathrm{keV}$ transitions. This clearly shows that the 1217, 1421, and 1776 transitions are in sequence. The equivalent mirrored spectra for ${ }^{45} \mathrm{Ti}$ are shown in Figs. 6(b) and 6(d).

One important result here is the obviously different intensity of the parity-changing decay(s) from the $J^{\pi}=\frac{5}{2}^{+}$state in ${ }^{45} \mathrm{~V}$ when compared with that of ${ }^{45} \mathrm{Ti}$. In ${ }^{45} \mathrm{Ti}$, this intensity is carried by the $707 \mathrm{keV}$ transition to the $J^{\pi}=\frac{3}{2}^{-}$state. This is shown in the inset to Fig. 1(a) and is a weak transition in comparison with the strong $743 \mathrm{keV}$ yrast transition. 


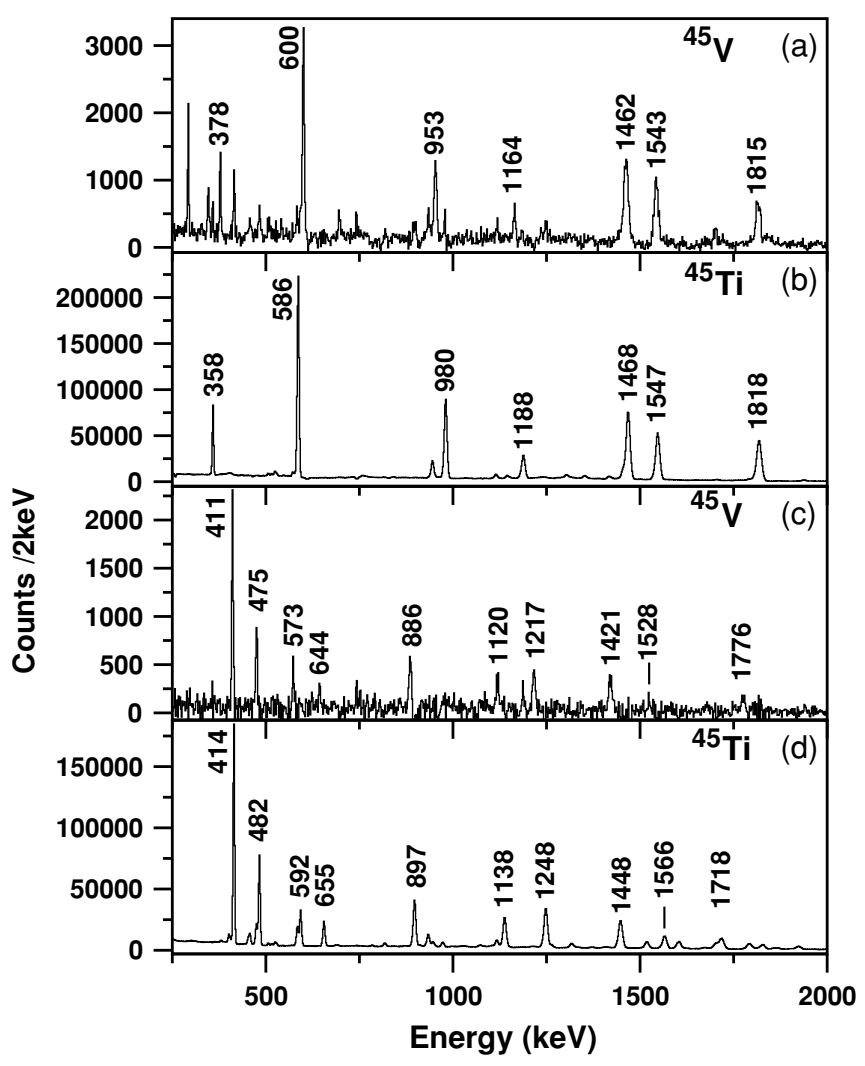

FIG. 4. Comparison of single-gated $\gamma$-ray spectra for the negative-parity bands [(a) and (b)] and the positive-parity bands [(c) and (d)] for the mirror pair, respectively, ${ }^{45} \mathrm{~V}$ and ${ }^{45} \mathrm{Ti}$. Spectra were taken from a coincidence matrix recorded when one neutron was detected and are in coincidence with (a) the $787 \mathrm{keV}$ transition in ${ }^{45} \mathrm{~V}$, (b) its mirrored transition, $743 \mathrm{keV}$, in ${ }^{45} \mathrm{Ti}$, (c) the $329 \mathrm{keV}$ transition in ${ }^{45} \mathrm{~V}$, and (d) its mirrored transition, $292 \mathrm{keV}$, in ${ }^{45} \mathrm{Ti}$.

In the inset of Fig. 1(d) the $741 \mathrm{keV}$ transition decaying from the analogous state in ${ }^{45} \mathrm{~V}$ can be seen and is about a factor of 10 stronger (see Table I) in relative intensity. In addition to the evidence presented in Figs. 1(a) and 1(d), the large difference in relative intensity between the 707 and $741 \mathrm{keV}$ analog transitions has also been confirmed in a triples analysis. Although it has not been possible to measure the proportions of the intensity flowing to the $J^{\pi}=\frac{5}{2}^{-}$and $\frac{3}{2}^{-}$states individually, the total intensity decaying from the $J^{\pi}=\frac{5}{2}^{+}$state is much larger in ${ }^{45} \mathrm{~V}$ than in ${ }^{45} \mathrm{Ti}$. Indeed, if the assignment of $J^{\pi}=\frac{5}{2}^{+} \rightarrow \frac{5}{2}^{-}$for the $741 \mathrm{keV}$ transition in Ref. [25] is correct, the equivalent transition in ${ }^{45} \mathrm{Ti}$ is either missing or extremely weak. Due to the strength of the $707 \mathrm{keV}$ transition in ${ }^{45} \mathrm{Ti}$ compared with the $414 \mathrm{keV} \mathrm{M1/E2}$ transition, strong contributions from multipoles of order 2 and higher are considered unlikely, and so we presume that this transition (and hence the mirrored $741 \mathrm{keV}$ transition in ${ }^{45} \mathrm{~V}$ ) is purely $E 1$ in character. The other two parity-changing transitions seen to de-excite the $J^{\pi}=\frac{7}{2}^{+}$state in ${ }^{45} \mathrm{Ti}$ (the 1226 and $1186 \mathrm{keV}$ transitions) are of low relative intensity (see Table I). The equivalent transitions in ${ }^{45} \mathrm{~V}$, if it is assumed

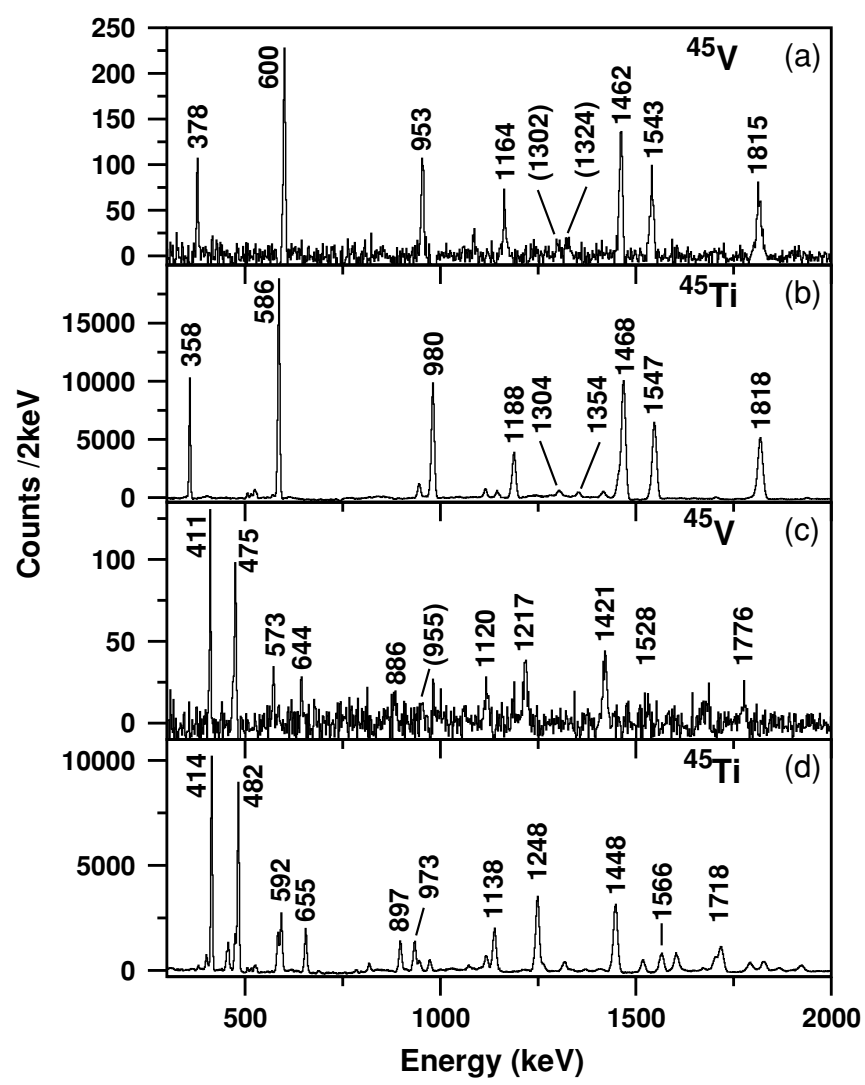

FIG. 5. Comparison of double-gated $\gamma$-ray spectra following a triples analysis for the negative-parity bands [(a) and (b)] and the positive-parity bands [(c) and (d)] for the mirror pair, respectively, ${ }^{45} \mathrm{~V}$ and ${ }^{45} \mathrm{Ti}$. Spectra are in coincidence with (a) the $787 \mathrm{keV}$ transition in ${ }^{45} \mathrm{~V}$ plus any of the 378,600 , or $953 \mathrm{keV}$ transitions, (b) the $743 \mathrm{keV}$ transition in ${ }^{45} \mathrm{Ti}$ plus any of the 358,586 , or $980 \mathrm{keV}$ transitions, (c) the $329 \mathrm{keV}$ transition in ${ }^{45} \mathrm{~V}$ plus any of the $411,475,886$, or $1217 \mathrm{keV}$ transitions, (d) the $292 \mathrm{keV}$ transition in ${ }^{45} \mathrm{Ti}$ plus any of the $414,482,897$, or $1248 \mathrm{keV}$ transitions.

that they carry the same intensity, would not be observed in the present work.

\section{DISCUSSION}

The different $E 1$ intensity is of considerable interest, as relative intensities between mirror pairs have previously been observed to be almost identical. Indeed, the two other cases of anomalous decay intensities that have recently been observed-for the $A=35$ mirror pair at low spin [33] and at high spin [34] and the $A=31[35,36]$ mirror pair-both involve $E 1$ transitions. In general, when considering the isospin dependence of transition strengths, it is found [37] that $E 1$ transitions, which proceed only through an isovector component of the transition operator, should have identical strengths in mirror partners. Indeed, this is not generally true for $E 2$ or $M 1$ transitions-although the expected approximate similarity in wave functions between mirror partners tends to result in similar decay branching ratios and hence similar relative intensities. It is therefore noteworthy that this intensity 


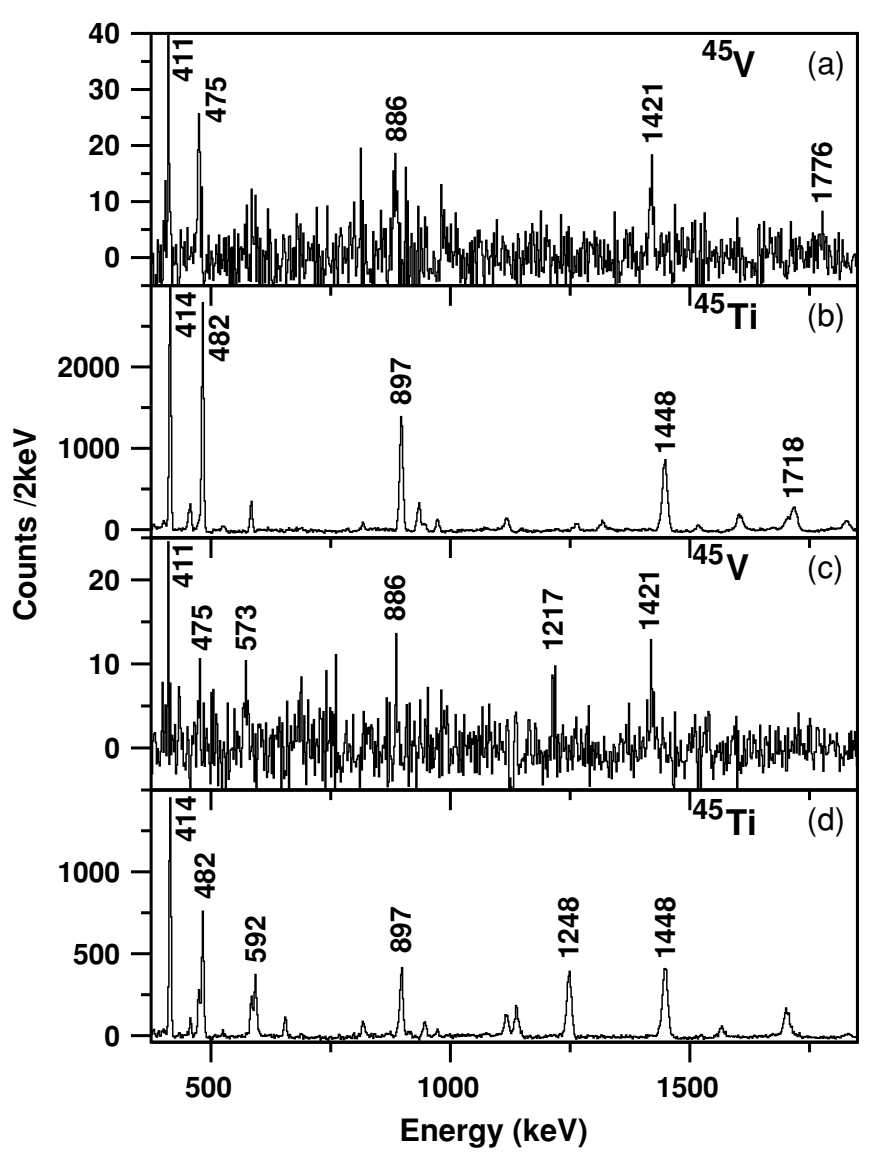

FIG. 6. Examples of double-gated $\gamma$-ray spectra following a triples analysis for the positive-parity bands in ${ }^{45} \mathrm{~V}$ [(a) and (c)] and ${ }^{45} \mathrm{Ti}$ [(b) and (d)]. Spectra are in coincidence with (a) the 329 and $1217 \mathrm{keV}$ transitions in ${ }^{45} \mathrm{~V}$, (b) the 292 and $1248 \mathrm{keV}$ transitions in ${ }^{45} \mathrm{Ti}$, (c) the 329 and $1776 \mathrm{keV}$ transitions in ${ }^{45} \mathrm{~V}$, (d) the 292 and $1718 \mathrm{keV}$ transitions in ${ }^{45} \mathrm{Ti}$.

anomaly has so far been observed only for $E 1$ transitions- the only type of decay where the transition strengths should be equal. Ekman et al. [33] suggested that this could result from isospin mixing, to which $E 1$ transitions may be particularly sensitive. Of course, the data presented so far consider only relative intensities and not transition strengths. This interpretation thus awaits measurement of transition strengths and, of course, detailed calculations.

The measured MED for the $f_{7 / 2}$ band, plotted as $E_{x}(J)\left[{ }^{45} \mathrm{~V}\right]-E_{x}(J)\left[{ }^{45} \mathrm{Ti}\right]$, is shown in Fig. 7. It is now well established (e.g. $[2,6,7,10]$ that the largest spin-dependent phenomenon contributing to Coulomb effects is the alignment or recoupling of pairs of protons from $J=0$ to $J=6-$ the maximum allowed for an $f_{7 / 2}$ pair. As the spin recouples, the spatial overlap of the pair is reduced, resulting in a reduction in the Coulomb energy. This effect is typically around $100 \mathrm{keV}$ for $f_{7 / 2}$ particles. The largest effect observed in the experimental MED plot is the rise from $\approx-30$ to $\approx+50 \mathrm{keV}$ from the lowest point around $J^{\pi}=\frac{13}{2}^{-}$to the peak at $J^{\pi}=\frac{19}{2}^{-}$. This can be understood easily in an $f_{7 / 2}$ picture in terms of the proton recoupling effect as follows. In

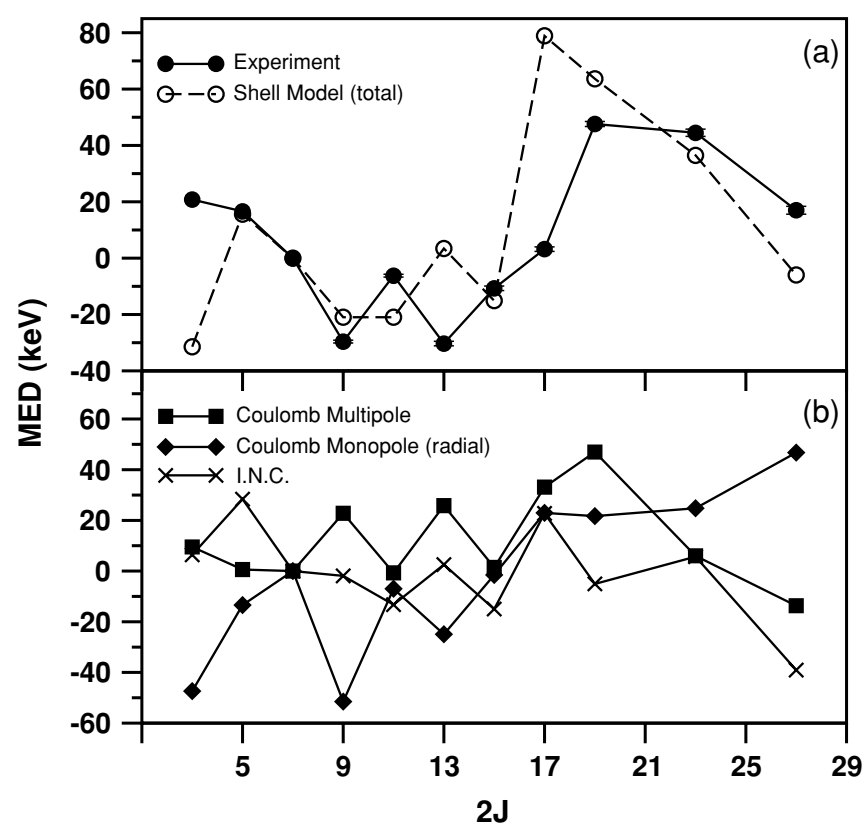

FIG. 7. Mirror energy differences-defined as $E_{x}(J)\left[{ }^{45} \mathrm{~V}\right]-$ $E_{x}(J)\left[{ }^{45} \mathrm{Ti}\right]$ - between states in ${ }^{45} \mathrm{~V}$ and ${ }^{45} \mathrm{Ti}$ for the negative-parity yrast sequence as a function of spin. (a) Comparison of the experimental MED with those predicted using the full $p f$-shell model. (b) The three components (see text) of the predicted MED, the sum of which give the model prediction in (a).

${ }^{45} \mathrm{Ti}$, the odd neutron blocks the alignment of a pair of neutrons, and so a pair of $f_{7 / 2}$ protons recouples, eventually to the full alignment of $J=6$. Indeed, one easily imagines the $J^{\pi}=\frac{19}{2}^{-}$ state in ${ }^{45} \mathrm{Ti}$ with a configuration similar to the $J^{\pi}=\frac{7}{2}^{-}$ ground state but with the two protons maximally aligned to $J=6$ rather than $J=0$ - i.e. with a significant contribution from the $\left[\pi\left(f_{7 / 2}\right)^{2}\right]^{J=6} \otimes\left[v\left(f_{7 / 2}\right)^{3}\right]^{J=7 / 2}$ configuration. The same is true for ${ }^{45} \mathrm{~V}$, but swapping protons for neutrons. Thus, the alignment of protons in ${ }^{45} \mathrm{Ti}$ (and hence neutrons in ${ }^{45} \mathrm{~V}$ ) results in the MED peak at $J^{\pi}=\frac{19}{2}^{-}$. As the $J^{\pi}=\frac{27}{2}^{-}$ band-terminating state is approached, the alignment of the other type of particle is required in each case. Thus the Coulomb effects go in the opposite direction, and the MED reduces back toward zero at high spins. It is interesting to note the overall downward trend of the MED at low spins, before the alignment effect. There is no obvious intuitive explanation for this, and this anomalous low-spin behavior has been seen in a number of other cases (e.g., $A=47$ and 49 [7]). It has been shown $[10,21]$ that an anomalously high two-body Coulomb matrix element for $J=2$ needs to be incorporated into the calculations to account for the experimental data. This is the so-called $J=2$ anomaly. It is expected that this anomaly should influence the MED at low spins, and the observed low-spin variations seen in MED could be attributed, at least in part, to this anomaly. The presence of the negative dip in the MED in this $A=45$ pair [Fig. 7(a)] from $J^{\pi}=\frac{3}{2}^{-}$to $J^{\pi}=\frac{13}{2}^{-}$is also consistent with the effect of this anomaly, although Fig. 7(b) indicates that the radial Coulomb effect (see below) is also important here. 
As with other studies in this shell, the large-scale $p f$-shell model has been used to help interpret these data. This model, based on the work of Caurier et al. [20], has been modified (e.g. $[7,15,21]$ to include different Coulomb phenomena and has to date been remarkably successful in giving a quantitative description of the trends of Coulomb effects with spin. In Fig. 7 we present the results of the latest version of the model, described by Zuker et al. [21]. In this case three components of the MED are determined separately as a function of spin. These components are presented individually in Fig. 7(b), the sum of which provide the total shell-model prediction of Fig. 7(a). Details of this can be found in Ref. [21], but the principles are outlined as follows. First, the Coulomb multipole term accounts for angular momentum recoupling of protons. It uses Coulomb matrix elements determined in a harmonic oscillator basis. Second, the Coulomb monopole term accounts for Coulomb effects associated with changes in radii as a function of spin. Third, the isospin nonconserving (INC) component is an additional multipole term added for $J=2$ proton couplings, the inclusion of which accounts for the $J=2$ anomaly described earlier. In other cases of mirror pairs higher in the shell, the agreement with this model has been extremely good (e.g. $[7,8,10])$. For this $A=45$ pair, however, the agreement in Fig. 7(a) is less good, and for some states (e.g., the $J^{\pi}=\frac{17}{2}^{-}$state) the model fails completely. Indeed, unlike in the heavier cases, there seems little to be learned from examination of the different components in Fig. 7(b). Similar difficulties were experienced [21] in describing the $A=46$ mirror pair. It is suggested that for these lighter systems the full $p f$ valence space is not appropriate and that perhaps two-particle two-hole excitations from the $s d$ orbits below ${ }^{40} \mathrm{Ca}$ need to be included.

It is of particular interest in this work to examine the MED for the $d_{3 / 2}$ band, which is shown in Fig. 8. Here the MED is plotted relative to the band-head energies of the corresponding $J^{\pi}=\frac{3}{2}^{+}$states. The MED shows a smooth decrease for most of the structure. It is possible to interpret this in a fairly

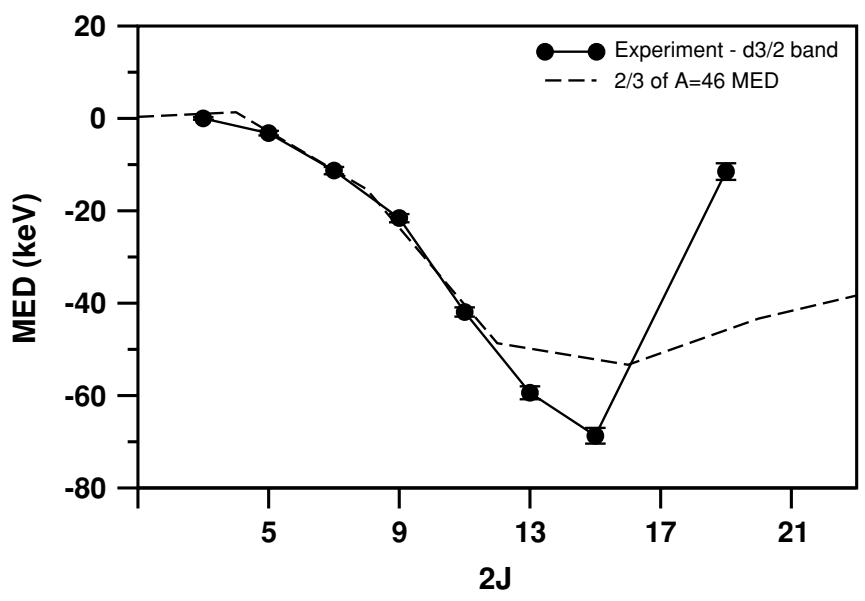

FIG. 8. Mirror energy differences between states in ${ }^{45} \mathrm{~V}$ and ${ }^{45} \mathrm{Ti}$ for the positive-parity sequence as a function of spin. The dashed curve shows the experimentally observed MED [12] for the $A=46$ mirror pair $\left({ }^{46} \mathrm{Cr} /{ }^{46} \mathrm{Ti}\right)$ multiplied by a factor of 0.66 (see text). straightforward way in terms of proton recoupling if it is assumed that (i) these structures correspond to a single $d_{3 / 2}$ hole in ${ }^{40} \mathrm{Ca}$ coupled to six $f_{7 / 2}$ valence particles and that (ii) the trends of the MED with spin are determined entirely by the $f_{7 / 2}$ particles - i.e., the $d_{3 / 2}$ hole is a spectator.

This analysis can be undertaken by carefully considering the possible structure of the $J^{\pi}=\frac{3}{2}^{+}$band-head state in ${ }^{45} \mathrm{Ti}$. The state can be formed either from a $d_{3 / 2}$ neutron excitation (i.e. $\left[\pi\left(f_{7 / 2}\right)^{2} v\left(f_{7 / 2}\right)^{4}\right]^{J=0} \otimes\left[v\left(d_{3 / 2}\right)^{-1}\right]^{J=3 / 2}$ or from a proton excitation $-\left[\pi\left(f_{7 / 2}\right)^{3} v\left(f_{7 / 2}\right)^{3}\right]^{J=0} \otimes\left[\pi\left(d_{3 / 2}\right)^{-1}\right]^{J=3 / 2}$. That is, the $T=\frac{1}{2} J^{\pi}=\frac{3}{2}^{+}$state in ${ }^{45} \mathrm{Ti}$ is constructed by coupling either a $d_{3 / 2}$ neutron-hole to a $T=1, J^{\pi}=$ $0^{+}$even-even core $\left({ }^{46} \mathrm{Ti}\right)$ or by coupling a $d_{3 / 2}$ protonhole to a $T=1, J^{\pi}=0^{+}$odd-odd core $\left({ }^{46} \mathrm{~V}\right)$. These two core states are, of course, isobaric analog states. Therefore, if we assume the approximations of perfect charge independence and pure isospin, we can determine the fractional contribution from each configuration from isospin coupling rules. The contribution depends on the square of the Clebsch-Gordan coefficient $\left(T^{\text {core }} T_{z}^{\text {core }}, t-t_{z} \mid T T_{z}\right.$ ), where $t$ and $t_{z}$ are the isospin and the isospin projection of the excited particle. The value of $(\mid)^{2}$ turns out to be $\frac{2}{3}$ for the former (neutron excitation) and $\frac{1}{3}$ for the latter (proton excitation). Following a similar argument, the $J^{\pi}=\frac{3}{2}^{+}$state in ${ }^{45} \mathrm{~V}$ is constructed by coupling either a $d_{3 / 2}$ proton-hole to a $T=1, J^{\pi}=0^{+}$ even-even core $\left({ }^{46} \mathrm{Cr}\right)$ or coupling a $d_{3 / 2}$ neutron-hole to a $T=1, J^{\pi}=0^{+}$odd-odd core $\left({ }^{46} \mathrm{~V}\right)$ - again with fractional contributions of $\frac{2}{3}$ and $\frac{1}{3}$. (Note, these fractions are also well reproduced from shell-model calculations). Hence it is easy to show that one might expect the trend of the the $A=45$ MED roughly to follow $\frac{2}{3}$ of the MED for the known [12] $T=1$ mirror pair ${ }^{46} \mathrm{Ti} /{ }^{46} \mathrm{Cr}$. This crude estimate is shown in Fig. 8 (dashed curve) for comparison and, although such a comparison on a state-by-state basis cannot be quantitatively meaningful, the agreement of the overall trend up to $J^{\pi}=\frac{11}{2}^{+}$ is impressive. To complete the story, it should be noted that these $T=\frac{1}{2}$ states can also be constructed by coupling a $d_{3 / 2}$ hole to a $T=0$ core (rather than $T=1$ ). However, $T=0$ restricts the core to $N=Z{ }^{46} \mathrm{~V}$, for which the lowest $T=0$ state is $J^{\pi}=3^{+}$. Thus, in both nuclei, this coupling would be expected to produce a $K^{\pi}=\frac{9}{2}^{+}$band head in addition to the $K^{\pi}=\frac{3}{2}^{+}$band, and for states with higher spins than $J^{\pi}=\frac{9}{2}^{+}$there would be two sets of states that could readily mix. Shell-model calculations performed including a $d_{3 / 2}$ excitation [38] suggest that these two sets of states do indeed mix. This provides an explanation for the deviation of the two curves at spins higher than $J^{\pi}=\frac{11}{2}^{+}$.

\section{SUMMARY}

A comprehensive new high-spin level scheme of the protonrich nucleus ${ }^{45} \mathrm{~V}$ including both negative-parity states built on $f_{7 / 2}$ configurations and a collective positive-parity structure built on a $d_{3 / 2}$ particle-hole excitation has been established. Comparisons with the mirror partner ${ }^{45} \mathrm{Ti}$ have been made, and the mirror energy, differences established and analyzed 
in terms of Coulomb phenomena. In particular, the MED of the structures built on the particle-hole excitation have been interpreted in terms of a core-hole coupling picture based on isospin coupling rules. The $E 1$ decay pattern observed in the mirror pair does not follow the same symmetry picture seen for the $M 1 / E 2$ transitions. This appears to be an emerging phenomenon that needs to be investigated both experimentally and theoretically.

\section{ACKNOWLEDGMENTS}

The authors particularly thank the accelerator staff at the Vivitron facility at IReS Strasbourg for their enormous efforts in producing the difficult ${ }^{24} \mathrm{Mg}$ beam needed for this run. This work was supported by the United Kingdom Engineering and Physics Sciences Research Council, the Swedish Research Council and the European Union through contract EUROVIV: HPRI-CT-1999-00078.
[1] R. Machleidt and H. Muther, Phys. Rev. C 63, 034005 (2001).

[2] J. A. Cameron, M. A. Bentley, A. M. Bruce, R. A. Cunningham, W. Gelletly, H. G. Price, J. Simpson, D. D. Warner, and A. N. James, Phys. Lett. B235, 239 (1990).

[3] J. A. Cameron, M. A. Bentley, A. M. Bruce, R. A. Cunningham, W. Gelletly, H. G. Price, J. Simpson, D. D. Warner, and A. N. James, Phys. Rev. C 44, 1882 (1991).

[4] J. A. Cameron, M. A. Bentley, A. M. Bruce, R. A. Cunningham, W. Gelletly, H. G. Price, J. Simpson, D. D. Warner, A. N. James, and P. Van Isacker, Phys. Lett. B319, 58 (1993).

[5] J. A. Cameron, M. A. Bentley, A. M. Bruce, R. A. Cunningham, W. Gelletly, H. G. Price, J. Simpson, D. D. Warner, and A. N. James, Phys. Rev. C 49, 1347 (1994).

[6] C. D. O’Leary, M. A. Bentley, D. E. Appelbe, D. M. Cullen, S. Erturk, R. A. Bark, A. Maj, and T. Saitoh, Phys. Rev. Lett. 79, 4349 (1997).

[7] M. A. Bentley, C. D. O'Leary, A. Poves, G. Martinez-Pinedo, D. E. Appelbe, R. A. Bark, D. M. Cullen, S. Erturk, and A. Maj, Phys. Lett. B437, 243 (1998).

[8] M. A. Bentley et al., Phys. Rev. C 62, 051303(R) (2000).

[9] J. Ekman et al., Eur. Phys. J. A 9, 13 (2000).

[10] S. J. Williams et al., Phys. Rev. C 68, 011301(R) (2003).

[11] D. Rudolph et al., Z. Phys. A 358, 379 (1997).

[12] P. E. Garrett et al., Phys. Rev. Lett. 87, 132502 (2001).

[13] S. M. Lenzi et al., Phys. Rev. C 60, 021303(R) (1999).

[14] C. D. O'Leary, M. A. Bentley, D. E. Appelbe, R. A. Bark, D. M. Cullen, S. Erturk, A. Maj, J. A. Sheikk, and D. D. Warner, Phys. Lett. B459, 73 (1999).

[15] S. M. Lenzi et al., Phys. Rev. Lett. 87, 122501 (2001).

[16] C. E. Svensson et al., Phys. Rev. C 58, R2621 (1998).

[17] C. D. O'Leary et al., Phys. Lett. B525, 49 (2002).

[18] A. Gadea et al., Legnaro National Laboratory Ann. Rep. 2003, INFN (REP) 202/2004, p. 8.
[19] J. A. Nolen and J. P. Schiffer, Annu. Rev. Nucl. Sci. 19, 471 (1969).

[20] E. Caurier, A. P. Zuker, A. Poves, and G. Martinez-Pinedo, Phys. Rev. C 50, 225 (1994).

[21] A. P. Zuker, S. M. Lenzi, G. Martinez-Pinedo, and A. Poves, Phys. Rev. Lett. 89, 142502 (2002).

[22] E. Caurier, J. L. Egido, G. Martinez-Pindo, A. Poves, J. Retamosa, L. M. Robledo, and A. P. Zuker, Phys. Rev. Lett. 75, 2466 (1995).

[23] J. A. Cameron et al., Phys. Rev. C 58, 808 (1998).

[24] F. Brandolini et al., Phys. Rev. C 60, 041305(R) (1999).

[25] S. A. Gronemeyer, L. Meyer-Schutzmeister, A. J. Elwyn, and G. Hardie, Phys. Rev. C 21, 1290 (1980).

[26] J. Simpson, Z. Phys. A 358, 139 (1997).

[27] F. A. Beck, Prog. Part. Nucl. Phys. 28, 443 (1992).

[28] A. Gadea et al., LNL Annual Report 1999, p. 151, INFN(REP) 160/00.

[29] O. Skeppstedt et al., Nucl. Instrum. Methods A 421, 531 (1999).

[30] P. Bednarczyk et al., Eur. Phys. J. A 2, 157 (1998).

[31] J. Ljungvall and M. Palacz, and J. Nyberg, Nucl. Instrum. Methods A 528, 741 (2004).

[32] P. Bednarczyk et al., Eur. Phys. J. A 20, 45 (2003).

[33] J. Ekman et al., Phys. Rev. Lett. 92, 132502 (2004).

[34] F. Della Vedova et al., Legnaro National Laboratory Ann. Rep. 2004, INFN (REP) 204/2004, p. 7.

[35] F. Della Vedova et al., Legnaro National Laboratory Ann. Rep. 2003, INFN (REP) 202/2004, p. 3.

[36] D. G. Jenkins et al., Phys. Rev. C 72, 031303(R) (2005).

[37] E. Warburton and J. Weneser, in Isospin in Nuclear Physics, edited by D. H. Wilkinson (North Holland, Amsterdam, 1969), Chap. 5.

[38] A. Poves (private communication). 